\title{
Escola de Química 2008 - Universidade do Porto
}

A $2^{a}$ edição da Escola de Verão em Química realizou-se no Departamento de Química (DQ) da Faculdade de Ciências da Universidade do Porto (UP), de 7 a 11 de Julho de 2008, integrada nas actividades da Universidade Júnior, que garantiu todo o apoio logístico (alojamento na Residência Professor Novais Barbosa e refeições nas cantinas dos Serviços Sociais).

Mais uma vez, tivemos o privilégio de receber vinte jovens formandos entre os quinze e os dezassete anos, com elevado nível intelectual e qualidades de trabalho excepcionais, o que, aliado ao generoso empenho do Departamento de Química em Ihes proporcionar boas condições de acolhimento, resultou numa semana muito bem sucedida para todos. to a dos Materiais, do Ambiente, da Saúde e da Energia.

O elevado número de candidatos à frequência da Escola (setenta e quatro candidaturas provenientes de todo o País!) e a motivação transmitida pelos mesmos, contrastando com o reduzido número de vagas, tornaram a tarefa de selecção difícil.

Estamos convictos, contudo, de que os jovens seleccionados aproveitaram a possibilidade de experimentar a procura de respostas a desafios químicos em que foi evidenciado o papel da ciência e/ou a tecnologia para o progresso e o desenvolvimento sustentável da Sociedade. Não duvidamos, também, que os nossos jovens investigadores, com o seu espírito criativo e o gosto pela inovação, se envolveram em cada problema com o entusiasmo necessário para o sentir.

Para o elevado nível da nossa Escola de Química muito contribuiram os responsáveis dos projectos e o apoio dos seus incansáveis colaboradores, que acompanharam os formandos ao longo da semana.

Este ano, o programa da Escola incluiu ainda duas lições proferidas pelos Professores Carlos Corrêa ("Química no Mundo Real") e António Fernando Silva ("Alguns Desafios da Química no Início do Século XXl"), que, desafiando a curiosidade dos jovens, contribuiram marcadamente para o aumento da motivação e interesse dos alunos pelas actividades que estavam a realizar.

De facto, a complementaridade destas apresentações, com abordagens diversas da Química, demonstraram como os Químicos, nos mais diversos sectores, contribuem (ou podem contribuir) para o desenvolvimento de uma Sociedade moderna e equilibrada.

No último dia da Escola foi realizado um Seminário Científico, com a participação de todos os formandos, que apresentaram oralmente 0 trabalho desenvolvido.

Formandos da Escola de Química 2008

Sob o lema "Participar, Experimentar, Sentir...", a Escola de Química 2008 teve como principal objectivo proporcionar a alunos do Ensino Secundário, que concluiram o $10^{\circ}$ (catorze alunos) ou $11^{\circ}$ anos (seis alunos), um estágio durante o qual puderam participar no desenvolvimento de um projecto em Química, integrados numa equipa de investigação.

A Escola permitiu evidenciar junto desses alunos as potencialidades da Química na resposta a desafios inovadores, em áreas tão diversas quan-

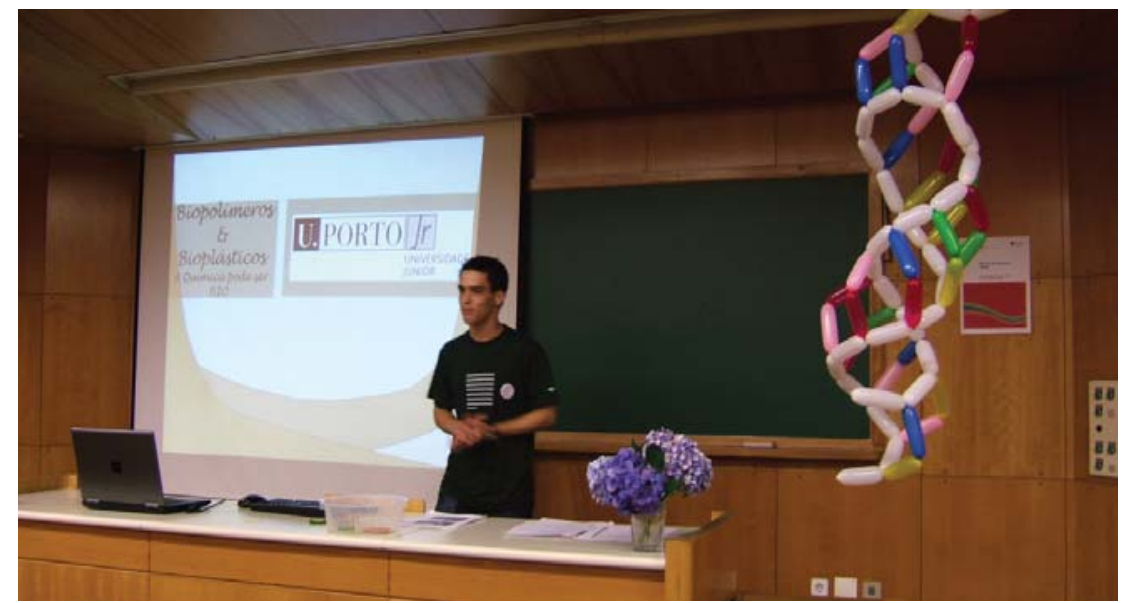

Apresentação de uma comunicação durante o Seminário 
Foi um momento muito gratificante para os membros do $D Q$, pois as exposições dos investigadores júniores tornavam difícil acreditar que os seus autores apenas se tinham dedicado às actividades durante cinco dias.

Para este sucesso não serão alheios também os professores do Ensino
Secundário que recomendaram excelentes formandos.

Com a organização desta Escola, o Departamento de Química da Faculdade de Ciências e a Reitoria da Universidade do Porto apostaram em dar mais um contributo para a divulgação da Ciência e para a formação de uma

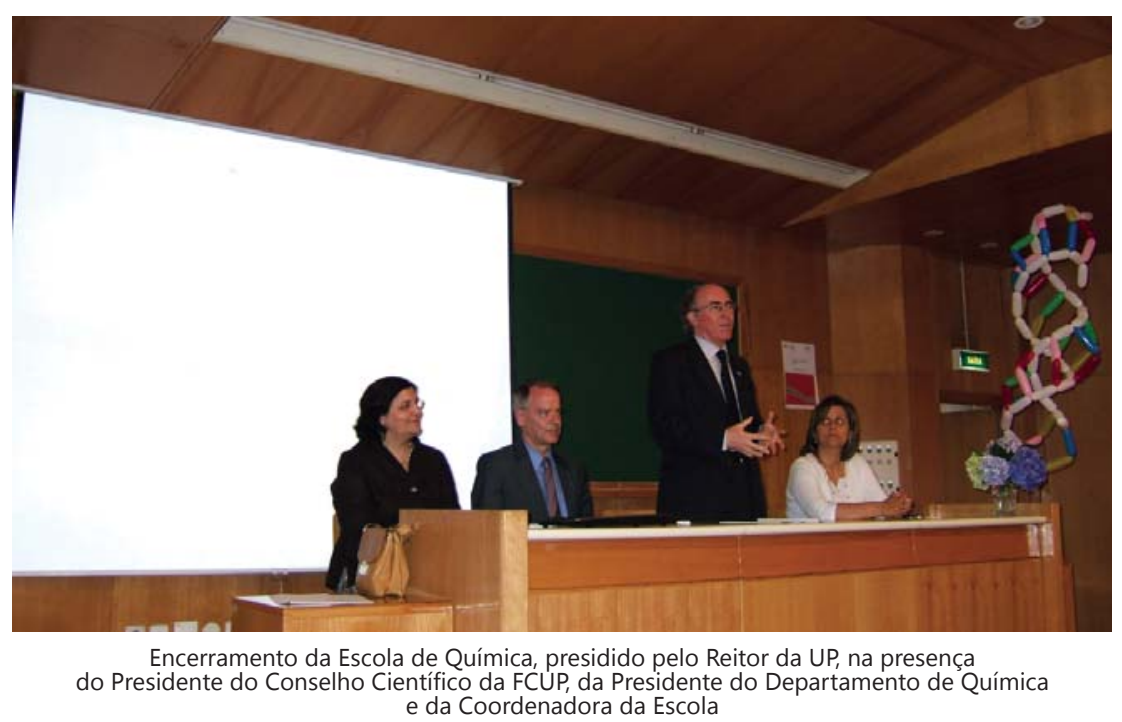

Sociedade mais atenta áquilo que a condiciona e, consequentemente, com maior capacidade crítica.

Um agradecimento é devido a todos os que de alguma forma tornaram viável mais esta iniciativa, incluindo a SPQ e a Porto Editora por atenderem às nossas solicitações.

O balanço muito positivo desta iniciativa deverá pesar como elemento fortemente dinamizador da realização de novas edições da Escola de Química UP!

Aos Investigadores Júniores, desejamos que a frequência desta Escola na Universidade do Porto os tenha incentivado a continuar cada vez mais atraídos pelos desafios da Ciência.

Parabéns pela participação!

Maria das Dores Ribeiro da Silva http://www.fc.up.pt/qui

\section{Actualidade Científica}

\section{UMA NOVA ESPERANÇA PARA OS DOENTES DE ALZHEIMER?}

A doença de Alzheimer é uma doença neurodegenerativa, constituindo a primeira causa de demência relacionada com a idade e que afecta cerca de 15 milhões de pessoas a nível mundial. Não foi ainda encontrada a forma de combater as suas causas.

Muitos anos antes da manifestação dos sintomas clínicos da doença, ocorre a formação de depósitos (placas) de um peptídeo conhecido como amilóide beta.

As descobertas mais recentes indicam que pequenos agregados solúveis do amilóide estão na origem da perda das funções aprendizagem e memória.

Estes oligómeros, com cerca de doze unidades peptídicas, têm um forte efeito tóxico sobre as células nervosas.
Um grupo de investigadores israelitas, coordenado por Ehud Gazit da Universidade de Telavive, desenvolveu uma nova molécula que combina dois aminoácidos e que tem por objectivo bloquear a formação dos oligómeros tóxicos (Angewandte Chemie International Edition 47 (2008) DOI: 10.1002/ anie.200802123).

O pequeno dipeptídeo desenvolvido por este grupo combina o ácido a-aminoisobutírico, que age com destruidor das camadas beta, com um segundo aminoácido, D-triptofano, que contém um grupo indole que se liga a grupos aromáticos, actuando este aminoácido ainda como estabilizador do dipeptídeo. Além disso, esta molécula é suficientemente pequena para poder ser absorvida a partir do sistema digestivo, não se degrada rapidamente no organismo e não é tóxica.

Os grupos aromáticos têm um papel importante na agregação dos peptí- deos que formam amilóides, pelo que esta nova molécula deve ligar-se ao centro aromático do amilóide beta através da sua componente aromática, inibindo a formação dos seus agregados tóxicos.

Este medicamento foi testado em ratos geneticamente modificados com doença de Alzheimer. Verificaram que eles recuperavam as suas funções cognitivas.

Além disso, verificaram ainda que ocorria uma drástica redução de amilóides e que o tamanho das placas de amilóides encontradas nos cérebros destes ratos também diminuía. 


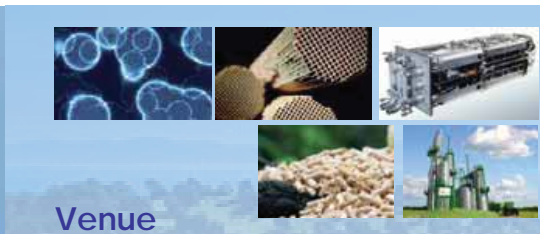

CLEAR summer school will be held at the Sithonia Thalasso \& SPA Hotel at the luxury Porto Camas Grand Resort The hotel is located on the westem coast of Sithonia, Chalkidiki's central peninsula in Northem Greece.

The Porto Carras Grand Resort is located $120 \mathrm{~km}$ from Thessaloniki, the second la rgest city in Greece, with an intemational airport.

A pick-up will be arranged at Macedonia Airport of Thessaloniki on Sunday May $24^{\text {th }}$ to transport the participants to the meeting venue.

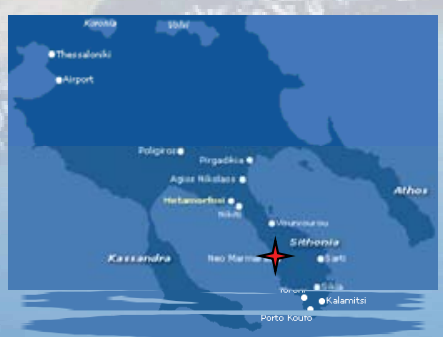

www.portocanas.com

\section{Sc ientific Committee}

Prof. la covos A. Vasalos, CPERI, Chair Prof. Gabriele Centi, IDECAT Prof. J osé Figueiredo, Univ. Porto Dr. Angelos Lappas, CPERI Prof. Roel Prins, EFCATS Prof. Ewa Serwicka, ACENET Prof. Kostas Triantafyllid is, AUTH Prof. Dimitrios Tsiplakides, $\mathrm{AUTH}$ Prof. Louis Vertegaal, ACENET

\section{Organizing Committee}

Prof. Angeliki Lemonidou (AUTH/CPERI) Dr. Stella Balomenou (CPERI)

Dr. Eleni Heracleous (CPERI)

Dr. Eleni lliopoulou (CPERI)

\section{Contact}

For further information please contact Dr. Eleni lliopoulou

朁 +302310498312

目 +302310498380

E-mail: eh@cperi.certh.gr

Web site

http:// www.clear.certh.gr

\section{EFCATS דCEIVI}

Summer School in Catalysis 2009

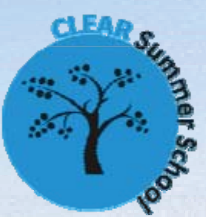

Catalysis

Lectures for Environmental Applications and Renewables

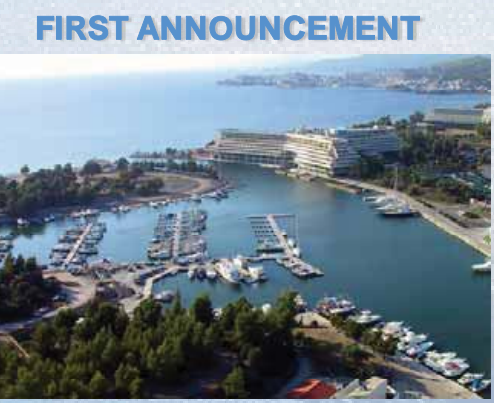

Porto Canas, Chalkidiki, Greece

May $24-29,2009$

Under the auspices of CERTH and IDECAT

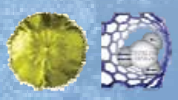

The European Federation of Catalysis Societies - EFCATS and the Applied Catalysis European Network - ACENET will hold the CLEAR summer school: "Catalysis Lectures for Environmental Applic ations and Renewables".

CLEAR Summer school aims in the effective training of young researchers on most up-to-date technological and research fields of applied catalysis, focusing on energy and environmentoriented topics.

The Summer School is a five-day event, comprised of invited lectures from distinguished speakers, poster presentations by young researchers and interactive workshops on research case studies.

\section{Preliminary program}

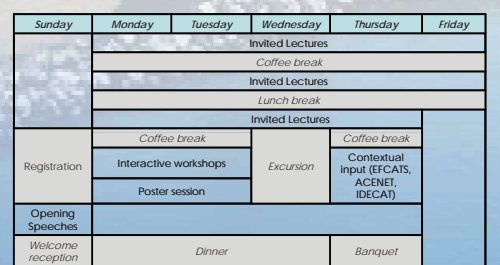

\section{Topics}

a Biomass utilization

a Biorefinery concept

a Thermochemical Processes for Liquid Biofuel Production

a Biotechnology/Enzymatic catalysis

a Homogeneous Catalysis

a Renewable Hydrogen Production

a Green Chemistry / Chemicals

a Novel Catalytic Materials

a Reaction Engineering

a Elec trochemistry/Fuel Cells

a Air/Water Pollution Abatement

a Clean Fossil Fuels

\section{Registration}

The registration fee includes full board with accommodation in a double-bed room from May $24-29$ and teaching material \& information package.

Academic participants.......

$400 €$

Industry participants.

$600 €$

\section{A number of grants will be available} for PhD students, covering the registration fee. Applic ations should be accompanied by a motivation letter and a recommendation letter from the supervisor.

\section{Abstracts}

The Organizing Committee invites young researchers to submit abstracts for poster presentations.

Participants are also encouraged to submit scientific challenges faced in the course of their research. The selected topics will be shortly presented and then discussed thoroughly during the interactive workshops. This action will be coordinated by the invited speakers relevant to the specific area of interest and aims to interaction, brainstoming and future collaborations between partic ipating research teams.

Abstracts should be submitted electronic ally using the Application Form available in the CLEAR website.

\section{Important dates \\ Opening date for abstracts $01 / 12 / 08$ \\ Closing date for abstracts $31 / 01 / 09$}

\section{Invited Speakers}

Prof. Robbie Burch, Queens Univ. Belfast, UK Prof. I ürgen Caro, Univ. Hannover, DE Prof. Gabrielle Centi, Univ. Messina, IT Prof. J osé Figueiredo, Univ. Porto, PT Prof. Dimitris Kondarides, U. Patras, GR Prof. Wa lter Leitner, RWTH Aachen, DE Prof. J ohannes Lercher, TUM, DE Prof. Guy Marin, UGent, BE Prof. Roel Prins, EIH Zurich, $\mathrm{CH}$ Prof. Costas G. Vayenas, U. Patras, GR Prof. Xenophon Verykios, U. Patras, GR 


\section{Actualidade Científica}

\section{Reacções Tubulares}

Recentemente, Dang Sheng Su e seus colegas do Fritz Haber Institute da Max Planck Society, em Berlim, Alemanha, anunciaram o desenvolvimento de uma forma de converter pequenas partículas altamente porosas de óxido de ferro provenientes de rochas vulcânicas em suportes para a deposição directa de nanotubos e nanofibras de carbono (Angew. Chem. 46 (2007) 1823-1824). O processo consiste na reacção directa de um gás orgânico na superfície das partículas vulcânicas. Agora, a equipa de investigação usou este catalisador de superfície modificada para activar um importante químico industrial, o butano, dispensando o recurso a um catalisador metálico bastante mais caro (Science 322 (2008) 73-77), possibilitando assim o novo processo uma alternativa económica ao processo clássico de activação de butano.

Su afirma que "Mostramos que quando os nanotubos de carbono são usados como catalisadores, podemos produzir alcenos de quatro carbonos sob condições muito selectivas, a baixa temperatura e baixa concentração de oxigénio, o que implica uma maior segurança." Acrescenta igualmente que "Durante os últimos dez anos, temos trabalhado continuamente com nanocarbono, mas todos os trabalhos prévios concentraram-se na activação de um composto aromático, o etilbenzeno. A activação de etilbenzeno a estireno é relativamente fácil de promover, já que o sistema é activado através do seu grupo aromático. O butano é muito menos reactivo e, assim, as selectividades elevadas observadas foram algo surpreendentes."

Deste modo, o processo alternativo proposto apresenta um grande potencial do ponto de vista ambiental, já que possibilita grandes poupanças de energia em relação aos processos estabelecidos. Su explica que "A desidrogenação oxidativa é um processo exotérmico que reduz consideravelmente as exigências energéticas do sistema. Por exemplo, o butadieno é industrialmente produzido através de steam cracking, que é um processo altamente endotérmico. A nova abordagem proposta implica a substituição de um processo que consome energia por outro que produz energia, o que o torna extremamente interessante do ponto de vista industrial."

A equipa optou por explorar a utilização de nanotubos de carbono numa perspectiva de catálise heterogénea, incorporando-os em partículas sólidas em oposição à sua aplicação em solução. Su afirma que "Os nanotubos de carbono têm uma menor densidade do que os agentes de diluição inertes, o que assegura uma recuperação fácil daqueles depois da sua utilização, através, por exemplo, de peneiração mecânica. Adicionalmente, os nanotubos de carbono não são alterados durante a reacção, podendo ser reutilizados quase indefinidamente, o que praticamente torna desprezável o seu custo inicial após um número elevado de aplicações sucessivas". (adaptado de webzine Reactive Reports 75 (2008)).

Paulo Brito

\section{Actualidade Científica}

\section{CSI Waco}

Investigadores da Baylor University em Waco, Texas, propuseram um método estatístico que processa medidas espectroscópicas de forma bastante expedita e que permite a estimativa da idade de restos esqueléticos de forma muito mais exacta e rápida do que anteriormente. Logo que o esqueleto é exposto, são escassas as técnicas disponíveis que permitam a estimativa do chamado intervalo post-mortem de forma exacta e rápida. O problema agrava-se no caso do local onde ocorre a decomposição ser quente e húmido, já que o processo de esqueletização ocorre mais rapidamente.

A equipa de Baylor, chefiada pelo químico Kenneth Busch, co-director do Center for Analytical Spectroscopy, explica que durante o processo de envelhecimento os ossos perdem água e as suas proteínas decompõem-se nos aminoácidos constituintes. A equi- pa monitoriza este processo usando uma técnica espectroscópica e aplica posteriormente um modelo estatístico de forma a correlacionar os espectros com o intervalo post-mortem. Os testes laboratoriais geraram modelos que apresentam erros mínimos de 4 dias para ossos de 90 dias de idade. Busch afirma que "Em condições laboratoriais controladas, o método parece bastante promissor. Logo que o modelo de regressão é construído a partir dos dados espectrais, a estimativa da idade pode ser realizada apenas em alguns minutos, em vez de horas ou dias."

A equipa de investigação usou 28 ossos diferentes de suínos com idades até aos 90 dias e recorreu a espectroscopia de reflexão difusa para a estimativa da data da morte. Esta técnica espectroscópica é muito sensível ao conteúdo proteico e à humidade dos espécimes. Para além disso, é uma técnica não destrutiva que dispensa a recolha de amostras dos ossos.

Os investigadores verificaram que o espectro de reflexão difusa dos ossos não seguia um comportamento linear em relação à sua idade. Assim, dividiram os dados em três conjuntos, que foram usados na construção de três modelos estatísticos para o processo de envelhecimento. Concluiram que este procedimento possibilitava uma redução adicional do erro de previsão. A combinação de duas abordagens um modelo de análise discriminante seguido de um modelo de regressão segmentada - possibilitou a optimização dos resultados.

Busch e seus colaboradores apresentaram a sua técnica e os respectivos resultados no encontro anual da $\mathrm{Fe}$ deration of Analytical Chemistry and Spectroscopy Societies em Outubro de 2008. (adaptado de webzine Reactive Reports 75 (2008)).

Paulo Brito 